\title{
ZENTRALE FACETTEN DER SPIRITUALITÄT DES DEUTSCHEN ORDENS IM SPIEGEL DER „ÄLTEREN HOCHMEISTERCHRONIK“
}

\section{Die Grundfesten des geistlichen Lebens: \\ EIN „JUNGES“ FORSCHUNGSGEBIET}

Drû dinc sint, die gruntvestene sint eines iegelichen geistlichen lebenes, unde sint geboten in dirre regelen. ${ }^{1}$ Mit diesen Worten leiten die Brüder des Deutschen Ordens ihre erste Regel ein und zielen gleich zu Beginn des umfassenden Regelcorpus darauf ab, ihrem Leben und Wirken ein im christlichen Glauben gründendes Fundament zu geben, eine Tendenz innerhalb ihrer Ordensregel, auf die auch schon zuvor die Invocatio der Dreifaltigkeit am Auftakt des vorhergehenden Regelprologs hindeutet. ${ }^{2}$ Folglich bildet die Spiritualität einen Kern des - modern gesprochen - „Anforderungsprofils“ an die Mitglieder des Deutschen Ordens. Doch wie genau sind die in der Ordensregel angedeuteten Grundfesten des geistlichen Lebens zu verstehen und gibt es in diesem Zusammenhang charakteristische Strömungen oder Tendenzen, die der Spiritualität des Deutschen Ordens im Mittelalter zueigen waren?

Schlägt man in einem älteren Lexikon zu theologischen Themen den Begriff, Spiritualität‘ nach, so erhält man beispielsweise im 1964 erschienenen „Lexikon für Theologie und Kirche" den unkommentierten Verweis auf das Lemma ,Frömmigkeit. ${ }^{3}$ Erst in der neueren Forschung, so auch in der Ausgabe des „Lexikons für Theologie und Kirche“" aus dem Jahr 2006 wird die Spiritualität

1 Die Statuten des Deutschen Ordens nach den ältesten Handschriften, hrsg. v. M. Perlbach, Halle/ Saale 1890 (unveränderter Nachdruck der Ausgabe: Hildesheim-New York 1975), S. 29.

2 Vgl. Die Statuten (wie Anm. 1), S. 22; G. Wiechert, Die Spiritualität des Deutschen Ordens in seiner mittelalterlichen Regel, in: Die Spiritualität der Ritterorden im Mittelalter, hrsg. v. Z. H. Nowak (Ordines militares. Colloquia Torunensia Historica VII), Toruń 1993, S. 134.

3 Vgl. Lexikon für Theologie und Kirche, hrsg. v. J. Höfer, K. Rahner, Bd. 9, Freiburg ${ }^{2}$ 1964, Sp. 975. 
eigenständig und in Abgrenzung von der Frömmigkeit behandelt. ${ }^{4}$ Diese stichprobenartige Annäherung zeigt die Problematik auf, die sich bei der Beschäftigung mit der hoch- und spätmittelalterlichen Frömmigkeitsgeschichte ergibt: Die systematische Erforschung von Spiritualität und Frömmigkeit kann auf keine weit zurückreichende Forschungstradition zurückblicken, so dass daher auch die Zahl der wissenschaftlichen Publikationen zu diesem Thema (im Gegensatz zu den zahlreichen esoterisch orientierten Handbüchern) sehr überschaubar ist. Was im Allgemeinen für die generelle Erforschung der Spiritualitäts- und Frömmigkeitsgeschichte gilt, ist ebenso im Speziellen auf die Forschungslage zur Spiritualität des Deutschen Ordens übertragbar. ${ }^{5}$ Zwar liegen mit den 1993 veröffentlichten Beiträgen des Thorner Kolloquiums „Die Spiritualität der Ritterorden im Mittelalter" aus dem Jahre 1991 sowie dem schon 1985 publizierten Vortrag von Ewald Volgger zur „Theologie und Spiritualität der Ordensstatuten aus dem 13. Jahrhundert" erste wissenschaftliche Annäherungen an das Themenfeld vor, dennoch bedarf es weiterer Forschung unter besonderer Berücksichtigung des Deutschen Ordens und seiner Historiographie. ${ }^{6}$ Einen Beitrag hierzu soll die vorliegende Arbeit leisten, die sich der Frage nach der Spiritualität des Deutschen Ordens widmet und ihre Erkenntnisse aus dem Text der „Älteren Hochmeisterchronik“ bezieht. Für diese Untersuchung wurden relevante Passagen der Älteren Hochmeisterchronik ausgewählt, die ein möglichst breites Spektrum der Ausübung der Spiritualität innerhalb des Deutschen Ordens widerspiegeln. Um eine Systematisierung der verschiedenen Facetten der Ordensspiritualität zu erarbeiten, wurden die in den Texten beschriebenen Facetten der Spiritualität einerseits dem kollektiven und andererseits dem privaten Sektor zugeordnet. ${ }^{7}$ Zur kollektiven Ausübung der Spiritualität zählen einerseits, schon aufgrund ihres eigenen Selbstverständnisses, die Liturgie sowie die christliche Allegorese der Kreuzzüge (beispielsweise im Baltikum) und des Hospitalsdienstes. Die private Artikulation der Spiritualität umfasst vor allem verschiedene in der "Älteren Hochmeisterchronik“" geschilderte Begebenheiten aus dem Bereich der christlichen Mystik.

4 Vgl. Lexikon für Theologie und Kirche, hrsg. v. W. Kasper u. a., Bd. 9, Freiburg/Breisgau ${ }^{32006,}$ Sp. $852-860$.

5 Vgl. Wiechert (wie Anm. 2), S. 132 f.; K. Elm, Die Spiritualität der geistlichen Ritterorden des Mittelalters. Forschungsstand und Forschungsprobleme, in: Die Spiritualität der Ritterorden (wie Anm. 2), S. 7.

6 Vgl. Die Spiritualität der Ritterorden (wie Am. 2); E. Volgger, Theologie und Spiritualität der Deutschordensstatuten (des „Ordensbuches") aus dem 13. Jahrbundert, in: Die Regeln des Deutschen Ordens in Geschichte und Gegenwart, hrsg. v. E. Volgger, Lana bei Meran 1985, S. 36-59.

7 Vgl. L. Hödl, Spiritualität, in: Lexikon des Mittelalters, Bd. 7, München 1995, Sp. 2124. 
Die Grenze zwischen diesen beiden Themenfeldern verläuft nicht trennscharf, beispielsweise haben sich in der Schilderung der "Älteren Hochmeisterchronik“ auch während eines Gottesdienstes, also während liturgischer Akte, übersinnliche Erscheinungen ereignet, die eher dem Bereich der Mystik zuzurechnen sind. Daher musste bei der Systematisierung dem inhaltlichen Kern des jeweiligen Berichtes nachgegangen werden, um eine möglicherweise vom Autor der Quelle beabsichtigte Intention herausarbeiten zu können. In der vorliegenden Studie werden die ausgewählten Abschnitte aus dem Text der „Älteren Hochmeisterchronik" zunächst inhaltlich zusammengefasst, anschließend jeweils in ihren Besonderheiten beschrieben und in einen kulturgeschichtlichen oder theologischen Zusammenhang gestellt. Dennoch soll das Hauptinteresse nicht der Theologie und den verschiedenen Unterdisziplinen (Liturgiewissenschaft, Dogmatik) gelten, sondern die zu bearbeitenden Passagen sollen auf ihre Aussage über den Deutschen Orden und die darin ausgeübten Facetten der Spiritualität befragt werden, so dass der Theologie hier eine eher hilfswissenschaftliche Rolle zukommt.

Im Rahmen dieses Aufsatzes ist es einerseits nicht möglich, eine auf den vorhandenen Quellen basierende, vollständige Darstellung der Spiritualität innerhalb des Deutschen Ordens im hohen und späten Mittelalter zu erarbeiten, andererseits soll die Studie über eine reine katalogartige Zusammenstellung und Präsentation der Facetten der Spiritualität und ihrer Ausprägungen im Spiegel der „Älteren Hochmeisterchronik“ hinausgehen. Hierzu werden nicht nur, wie bereits erwähnt, die jeweiligen Passagen analysiert, die Rückschlüsse über die Spiritualität innerhalb des Deutschen Ordens zulassen, sondern es soll ebenso der jeweilige Kontext dieser Berichte untersucht werden, um eine mögliche Intention für die Schilderung innerhalb der Chronik herausarbeiten zu können.

Im Folgenden soll zunächst der Begriff der ,Spiritualität' möglichst präzise umrissen werden, woraus sich erste Anhaltspunkte für die Auswahl der Textstellen ergeben. Daran schließen sich die inhaltliche Darstellung der spirituellen Artikulationsformen innerhalb des Deutschen Ordens an. Den Abschluss bilden im Rahmen eines zusammenfassenden Ausblicks die Kontextualisierung der Spiritualitätsformen in der „Älteren Hochmeisterchronik“ und die Interpretation der Textstellen hinsichtlich einer Aussageabsicht oder Instrumentalisierung dieser Schilderungen.

\section{Christliche Spiritualität: Dimensionen eines Begriffs}

In Abgrenzung zur theoretischen Grundlegung des Christentums innerhalb der systematischen Theologie (beispielsweise in der Dogmatik) werden unter der Spi- 
ritualität praktische und daher auf Handlungen basierende Ausprägungen des religiösen Alltagslebens und der Vollzug verschiedener Glaubensinhalte verstanden. ${ }^{8}$ Hierzu zählen, neben dem Kult und der Liturgie, unter anderem private Formen wie das Gebet, Mystik und Askese. ${ }^{9}$ Zwar zielt in etymologischer Hinsicht die aus dem lateinischen Wort ,spiritus' (= ,Hauch', ,Atem', ,Geist') abgeleitete Spiritualität auf eine rein geistige oder emotionale Äußerung, doch ist die Spiritualität im traditionellen Verständnis in den Kontext der Beziehung des Menschen zu Gott einzuordnen, die sich durch spirituelle Übungen oder Handlungen ausdrücken kann. ${ }^{10}$ In Abgrenzung zur kollektiv verstandenen Spiritualität als Gesamtheit der Ausdrucksfacetten des geistlichen Lebens, umfasst der Begriff der ,Frömmigkeit' eher die private Geisteshaltung der respektvollen Ehrfurcht und Demut Gott gegenüber, die wiederum zu den Ausprägungen der Spiritualität gezählt werden kann. ${ }^{11}$ Dennoch ist eine Abgrenzung der beiden Begriffe voneinander sehr kompliziert, da sie im Sprachgebrauch meistens synonym verwandt werden.

Während die monastischen und klerikalen Kulthandlungen im Kollektiv der Gläubigen und ihre liturgischen Abläufe genauestens durch Rubriken geordnet und reglementiert sind und dabei einer kirchlichen Kontrolle unterliegen, ist die Ausübung der individuellen Andachtspraktiken zumeist nicht dezidiert fest- und vorgeschrieben. Dennoch können einzelne ritualisierte Abläufe wiederum (durch Rubriken oder eine inhaltliche Abfolge) strukturiert sein, wie zum Beispiel die Übungen des „Schatzbehalters“ von Stephan Fridolin, eines spätmittelalterlichen Andachtsbuches aus dem Jahre 1491. ${ }^{12}$ Einen Gegensatz zur flexiblen Handhabung der Andachtsformen bilden die Ordensstatuten, die den Ordensangehörigen bestimmte Gebete oder asketische Übungen vorschreiben können. ${ }^{13}$ Auch innerhalb des Deutschen Ordens existierte der Brauch, bestimmte Gebete zu sprechen, so beispielsweise das Stoßgebet Nos cum prole pia benedicat virgo Maria, das im Anschluss an jede Messfeier von den Brüdern aus Gewohnheit gemeinschaft-

8 Vgl. Wiechert (wie Anm. 2), S. 133.

9 Vgl. H. Waldenfels, Spiritualität. II. Religionswissenschaftlich, in: Lexikon für Theologie und Kirche (wie Anm. 4), Sp. 853.

10 Vgl. A. Bucher, Psychologie der Spiritualität, Weinheim, Basel 2007, S. 28.

11 Vgl. H. Frankemölle, Frömmigkeit. I. Biblisch u. Judentum, in: Lexikon für Theologie und Kirche (wie Anm. 4), Bd. 4, Freiburg im Breisgau 2006, Sp. 166.

12 Vgl. P. Seegets, Passionstheologie und Passionsfrömmigkeit im ausgehenden Mittelalter. Der Nürnberger Franziskaner Stephan Fridolin ( 1 1498) zwischen Kloster und Stadt, Tübingen 1998.

13 Vgl. B.-M, Rosenberg, Marienlob im Deutschordenslande Preußen. Beiträge zur Geschichte der Marienverehrung im Deutschen Orden bis zum Jahre 1525, in: Acht Jahrhunderte Deutscher Orden in Einzeldarstellungen. Festschrift zu Ehren Sr. Exzellenz P. Dr. Marian Tumler O. T. anlässlich seines 80. Geburtstages, hrsg. v. K. Wieser (Quellen und Studien zur Geschichte des Deutschen Ordens 1), Bad Godesberg 1967, S. 324. 
lich gebetet und in den Gesetzen des Hochmeisters Pauls von Russdorf schließlich 1422 fest verankert wurde. ${ }^{14}$

Bis in die heutige Zeit ist das Repertoire der verschiedenen Ausübungsformen christlicher Spiritualität einem stetigen Wandel unterworfen: Neue Andachtsformen bildeten sich im Laufe der Zeit aus, einst populäre Praktiken gerieten beinahe völlig in Vergessenheit, so auch zahlreiche Aspekte der Ausübung mittelalterlicher Spiritualität, die den thematischen Kern dieser Hausarbeit bilden. ${ }^{15}$ Während im Mittelalter die Spiritualität zumeist auf die kirchlich kanalisierte Frömmigkeits-praxis festgelegt war, ist sie heute oftmals von traditionellen Formen abgekoppelt und artikuliert sich nicht mehr nur innerhalb kirchlicher Strukturen, sondern in weltanschaulichen Strömungen, so der New-Age-Szene oder der ökologischen Bewegung. ${ }^{16}$

Jedoch ist eine bei diesen zeitgenössischen Gruppen getroffene Feststellung auch auf das Mittelalter und damit auf den Deutschen Orden anwendbar: Da sich die Spiritualität im individuellen Lebensvollzug und in der persönlichen Mentalität artikuliert, existieren so viele verschiedene Spiritualitäten, wie es spirituelle Menschen gibt. ${ }^{17}$ Dies bedeutet auf den Deutschen Orden übertragen: Es zeigen sich in der Schilderung der „Älteren Hochmeisterchronik“ Formen der Spiritualitätsausübung einzelner Mitglieder des Deutschen Ordens, die generelle Rückschlüsse über die Situation im Deutschen Orden zulassen. Allerdings bedeutet dies auch im Umkehrschluss, dass eine umfassend gültige Aussage über die Spiritualität des Deutschen Ordens nicht getätigt werden kann. Vielmehr zeigen sich verschiedene Facetten der Ordensspiritualität, deren Ausübung jedoch von Ordensmitglied zu Ordensmitglied variieren konnte, sofern sie nicht durch die Statuten verbindlich vorgeschrieben waren. Überlegungen zu diesem Themenfeld stehen im Mittelpunkt des sich nun anschließenden Kapitels, das ausgewählte Stellen der „Älteren Hochmeisterchronik“ hinsichtlich dieser Gesichtspunkte analysiert.

14 Vgl. Die Statuten (wie Anm. 1), S. 158: Statuerunt eciam, ut omnes sacerdotes ordinis post celebracionem missarum in ewangelio sancti Ioannis, dum sacerdos dicat: »et verbum caro factum est«, quod omnes fratres iacebunt in venia usque dicitur: »nos cum prole pia«.

$15 \mathrm{Zu}$ den Ausprägungsformen der christlichen Spiritualität im Wandel der Zeit vgl. J. Weismayer, Spiritualität. III. Historisch-theologisch, in: Lexikon für Theologie und Kirche (wie Anm. 4), Bd. 9, Sp. 853-856; Elm (wie Anm. 5), S. 11.

16 Vgl. Bucher (wie Anm. 10), S. 22.

17 Vgl. J. Sudbrack, Spiritualität. V. Typologien, in: Lexikon für Theologie und Kirche (wie Anm. 4), Bd. 9, Sp. 857; Wiechert (wie Anm. 2), S. 133; Elm (wie Anm. 5), S. 10. In dieser Hinsicht lässt sich auch eine Aussage Joseph Ratzingers verstehen, der auf die Frage seines Interviewpartners Peter Seewald, wie viele Wege zu Gott existieren würden, entgegnet, dass es so viele Wege geben würde, wie es Menschen gibt. Vgl. J. Ratzinger, Salz der Erde. Christentum und katholische Kirche im neuen Jahrtausend. Ein Gespräch mit Peter Seewald, München ${ }^{6} 2004$, S. 8. 


\section{FaCETten DeR kOlLekTiV Verstandenen Und AusgeübTen Spiritualität des Deutschen Ordens in Der „ÄLteren HOCHMEISTERCHRONIK“}

In der Schilderung der „Älteren Hochmeisterchronik“ überwiegen deutlich diejenigen Begebenheiten, die von übersinnlichen Erscheinungen, beispielsweise während des persönlichen Gebetes, und von Andachts- sowie Bußübungen handeln. Somit lassen sich diese Ereignisse gemäß der oben getroffenen Differenzierung in den Bereich der Mystik einordnen. Die Liturgie als gemeinschaftlich gefeierter Gottesdienst (Messfeier, Stundenliturgie) tritt in der Erzählung der Chronik eher in den Hintergrund. Dennoch können aus dem Erzählzusammenhang die den Alltag strukturierenden liturgischen Feiern ansatzweise erschlossen werden. Ein umfassenderes Bild ergibt sich jedoch, wenn auch die Statuten des Deutschen Ordens bei der Bearbeitung der Quelle ergänzend hinzugezogen werden. Im Gegensatz zur Liturgie als Form der kollektiven Ausübung der Spiritualität werden hingegen zahlreiche Feld- und Kreuzzüge gegen die Heiden als allegorisierter Dienst für den Glauben geschildert. Die sich nun anschließende Zusammenstellung und Analyse der Spiritualität innerhalb des Deutschen Ordens beginnt mit dem Themenkomplex der gemeinschaftlich praktizierten Spiritualität, auf die der in der Chronik ausführlicher behandelte Bereich der (privat ausgeübten) Mystik folgt.

\section{Liturgie}

Es sind vor allem zwei Textpassagen der „Älteren Hochmeisterchronik“, die Rückschlüsse auf die Liturgie des Deutschen Ordens im Hoch- und Spätmittelalter zulassen: Hierzu zählen die Schilderung der Ordensprofess Konrads von Thüringen im 44. und der Bericht eines samländischen Weisen im 46. Kapitel der Chronik. Aufgrund des historisch belegten Datums der Profess und der im Text genannten Jahreszahl eines Ereignisses nach dem Besuch des Weisen beim Deutschen Orden, lassen sich diese zwei Begebenheiten in die Jahre zwischen 1234 (Jahr der Profess Konrads) und 1254 einordnen. Zwar liegt der Fokus in der Beschreibung der Profess Konrads nach seiner Bekehrung und Berufung in den Ordensstand auf einem Erscheinungsbericht, doch es werden Riten während des Gottesdienstes - wenn auch nur kurz - genannt: Wesentliche Bestandteile der Liturgie zur Aufnahme in den Orden waren die Prostratio, das Sich-Niederlegen vor dem Altar, das Aufnahme- und Segensgebet und der Gesang des epikletischen Hymnus Veni Sancte Spiritus. ${ }^{18}$ Schon im frühen Mittelalter hatten sich diese Riten und Gebete sowie ihre

18 Vgl. Die Ältere Hochmeisterchronik, hrsg. v. M. Toeppen, in: Scriptores rerum Prussicarum. Die Geschichtsquellen der Preussischen Vorzeit bis zum Untergange der Ordensherrschaft, Bd. III, 
Abfolge etabliert und wurden im Laufe der Zeit innerhalb der verschiedenen Versionen des Pontificale auch für die Ordensprofess vorgeschrieben. ${ }^{19}$ Hinsichtlich der Spiritualität drückt die Prostratio in christlicher Anschauung die Unterwerfung der Professen unter den Willen der Kirche, ihre vollständige Indienstnahme durch Gott und besonders die Niedrigkeit des Menschen gegenüber der Größe Gottes aus. ${ }^{20}$ Laut Joseph Ratzinger ist das Sich-Niederwerfen vor dem Altar bei einer Weihe oder einer Profess der äußerlich sichtbare Ausdruck der „Unfähigkeit, aus Eigenem den [...] Auftrag Jesu Christi wahrzunehmen “21, was durch die Anrufung der Heiligen um ihren Beistand in der Allerheiligenlitanei noch unterstrichen wird. ${ }^{22}$ Die Prostratio drückt folglich gestisch eine innere Haltung aus, die vom Professkandidaten erwartet wird, daher auch vorgeschrieben ist, und sein geistliches Leben im Anschluss an die Aufnahme in den Orden bestimmen soll. Die Hilfe zur Ausübung dieser geistlichen Lebensführung wurde darüber hinaus bei der beschriebenen Profess (und wird bis in die heutige Zeit) durch das Segensgebet erbeten, bei Konrad von Thüringen und seinen Mitbrüdern wurde es sogar noch durch die Pfingstsequenz Veni Sancte Spiritus unterstrichen, welche den Heiligen Geist auf die Professkandidaten herabruft. ${ }^{23}$ An dieser Stelle erscheint es als sinnvoll, in aller Kürze die sich während der Profess ereignete Erscheinung zu beschreiben: Während die Professkandidaten ausgestreckt vor dem Altar lagen, konnten die Gottesdienstteilnehmer beobachten, wie sich die Feuerzungen des Heiligen Geistes auf Konrad und seine Mitbrüder herabsenkten. ${ }^{24}$ Das in der Profess in Erscheinung getretene besondere Charisma des Landgrafen zeigte sich auch darin, dass er die Sünden der ihm begegnenden Menschen intuitiv erkennen konnte und nur dann mit ihnen in Kontakt treten wollten, wenn sie gebeichtet hatten. ${ }^{25}$ Daraus lässt sich schließen, dass das geschickte Wirken Konrads als Hochmeister

hrsg. v. T. Hirsch, M. Toeppen, E. Strehlke, Leipzig 1866, S. 559: Do sy gestrackt vor dem altar login und eyn prister obyr en des ordens seyn las, und anhub zcu singen: »Veni sacte spiritus « [...].

19 Vgl. M. Kunzler, Prostration, in: Lexikon für Theologie und Kirche (wie Anm. 8), Bd. 8, Freiburg im Breisgau 2006, Sp. 648.

20 Vgl. J. Ratzinger (Benedikt XVI.), Der Geist der Liturgie. Eine Einführung, Freiburg im Breisgau 2006, S. 159-167; R. Suntrup, Die Bedeutung der liturgischen Gebärden und Bewegungen in lateinischen und deutschen Auslegungen des 9. bis 13. Jahrbunderts, München 1978, S. 166; J. Lechner, Liturgik des römischen Ritus, Freiburg 1953, S. 73.

21 Ratzinger (wie Anm. 20), S. 162.

22 Vgl. ebd., S. $161 \mathrm{f}$.

23 Vgl. Zeremoniale für die Bischöfe in den katholischen Bistümern des deutschen Sprachgebietes, Freiburg 1998, S. 206 f.

24 Vgl. Die Ältere Hochmeisterchronik (wie Anm. 18), S. 559: [...] do wart von al der umstendigen schar gesehin obyr en als eyn vuyrsflam, yn dem sy der heilige geist besas und sundirlich den lantgreven $[\ldots]$.

25 Vgl. ebd., S. 559. 
einerseits auf eine besondere Nähe zu Gott und seine Beseelung durch den Heiligen Geist zurückgeführt wird, dass er aber auch andererseits in der „Älteren Hochmeisterchronik" und ihrer Vorläufer als geistliches und politisches Vorbild für die gegenwärtigen Hochmeister angesehen wurde. Dieses Faktum ist insofern von besonderem Interesse, da Konrad von Thüringen bereits sechs Jahre nach seinem Ordenseintritt und nur ein Jahr nach der Übernahme des Hochmeisteramtes als Nachfolger Hermann von Salzas 1240 starb. ${ }^{26}$ Möglicherweise spiegelt die Darstellung Konrads innerhalb der Chronik seine Hochschätzung durch die jeweiligen Chronisten ${ }^{27}$ wider, die dadurch auch die großen Hoffnungen (geradezu in dramatischer Absicht) nachzuzeichnen versuchten, die auf Konrad gesetzt wurden, sich dann aber wegen des frühen Todes nicht realisieren ließen.

Im liturgischen Kontext ist außerdem der ebenfalls nur kurze Bericht des samländischen Weisen, den er seinen Landsleuten nach dem freundlichen Empfang durch die Brüder in einer Ordensburg erstattet, interessant, da er liturgische Abläufe aus einem „heidnischen“ und in die Chronik integrierten Blickwinkel wiedergibt. Bei seinem Besuch führten ihn die Ordensbrüder nicht nur durch capelle, remthere und schlafhusze $e^{28}$, sondern er nahm offensichtlich auch am Chorgebet teil oder bemerkte zumindest, dass sich die Ordensbrüder zu verschiedenen Tages- und Nachtzeiten in der Kirche zum Gottesdienst zusammenfanden. ${ }^{29}$ Recht beeindruckt schilderte er die Ordensdisziplin, insbesondere dass die Brüder sogar nachts aufstanden und in der Kirche Stundengebet hielten. Darüber hinaus war sich der weise Samländer dessen bewusst, dass seine Landsleute von Deutschordenstruppen angegriffen wurden, da sie eben nicht den christlichen Gott verehrten. ${ }^{30}$

Diese beiden Textstücke zur liturgisch ausgedrückten Spiritualität innerhalb des Ordens geben keine weiteren Informationen zu diesem Themenfeld. Daher müssen die Statuten des Deutschen Ordens zu liturgischen Gesichtspunkten näher befragt werden: An einer recht zentralen Stelle, schon in der 8. Ordensregel, wird der Gottesdienst der Ordensbrüder genauestens geregelt. Die Ordensangehörigen, sowohl die Priesterbrüder, als auch die Laien, sollten sich tages unde nahtes zu Gotes dieneste unde zu ir gezîten ${ }^{31}$ zusammenfinden, wobei an anderer Stelle

26 Vgl. J. Sarnowsky, Der Deutsche Orden, München 2007, S. 119.

27 Es handelt sich bei den besagten Chronisten einerseits um Peter von Dusburg, andererseits um Nikolaus von Jeroschin und die Verfasser der „Älteren Hochmeisterchronik“, die auf die Chronik Peter von Dusburgs (in diesem Abschnitt der Quelle) zurückgreifen konnten.

28 Die Ältere Hochmeisterchronik (wie Anm. 18), S. 560.

29 Vgl. ebd., S. 560: Des nachtis sten sy uf und gen zcu sammene yn yr bethus und des tagis manchstunt yrbitin sy ynniclich lob erem gote.

30 Vgl. ebd., S. 560: Daz thu wyr nicht. Dorum vorwar gesegen sy uns an.

31 Die Statuten (wie Anm. 1), S. 34. 
zwischen den Klerikern und Laien unterschieden wurde: Während die Priesterbrüder ihr Brevier, also das kirchlich vorgeschriebene und universale Stundengebet verrichten mussten, wurde den Laienbrüdern aufgetragen, in derselben Zeit eine je nach Horen unterschiedliche Anzahl von Pater noster zu beten. ${ }^{32}$ In dieser Differenzierung zeigt sich auch das Selbstverständnis der geistlichen Ritter: Da sie keine höheren Weihen empfingen, waren die Ritter zwar Kleriker (im Sinne von Angehörigen des geistlichen Standes), ihnen wurden jedoch keine Weiheämter übertragen, wie es bei Mönchen oder Stiftsherren der Fall war. Daher stand im Mittelpunkt ihres Ordenslebens nicht die Kontemplation oder die Liturgie, sondern die Militia Christi, der Kampf gegen die Glaubensfeinde; sie waren darüber hinaus auch nicht zum Breviergebet verpflichtet. ${ }^{33}$ Wenn es sich bei den Laien aber um gelehrte Ordensangehörige handelte, so sollten sie mit Erlaubnis des Oberen anstelle der einfacheren Vaterunser das Breviergebet mit den Priestern verrichten. ${ }^{34}$ Interessanterweise wurden nicht nur die sieben kanonischen Horen, sondern auch die besonders im Norden Europas weit verbreiteten „Marientiden“ verrichtet, die mit ihren Texten (Psalmen, Antiphonen, Lesungen) besonders auf Maria ausgerichtet waren und im Laufe der Zeit von einem Nebenoffizium zu einem eigenständigen Stundengebet avancierten. ${ }^{35}$ Hierin zeigt sich auch die besondere marianische Frömmigkeit des Ordens, auf die an einer späteren Stelle noch gesondert eingegangen wird.

Ebenfalls zum liturgischen Bereich und damit zur kollektiven Ausübung der Spiritualität zählt die Kommunionfrömmigkeit, die in der 9. Regel des Deutschen Ordens präzisiert wird: Im Verweis auf die Brotrede Jesu aus dem Johannesevangelium (Joh 6, 22-59) und den davon abgeleiteten und für das Seelenheil notwendigen regelmäßigen Kommunionempfang schrieb die Regel die Kommunion an sieben Tagen des Jahres vor, so an den kirchlichen Hochfesten (Gründonnerstag, Ostern, Pfingsten, Weihnachten) sowie an besonderen Marienfesten (Maria Lichtmess, Maria Himmelfahrt). ${ }^{36}$ Dass die Kommunion einerseits aufgrund der Vorschrift in der Ordensregel, andererseits auch durch den begierdehaften Wunsch nach dem Kommunionempfang an den festgelegten Tagen unbedingt zu sich ge-

32 Vgl. ebd., S. 34 f.; U. Horst, Die Statuten des Deutschen Ordens und die Konstitutionen der Dominikaner, Zeitschrift für die Geschichte und Altertumskunde Ermlands 30 (1962), 2, S. 361.

33 Vgl. Elm (wie Anm. 5), S. 20.

34 Die Statuten (wie Anm. 1), S. 35: [...] unde wanne der leigen brûdere genûge gelêret sint, swelcher von im selber oder mit urlobe des obersten mit den phaffen die tagecit oder die gezît von unser vrowen an den salmen unde an anderen dingen, die zu dem ambehte gehôrent der phaffen, sprechen wollent [...].

35 Vgl. Sarnowsky (wie Anm. 26), S. 77.

36 Vgl. Die Statuten (wie Anm. 1), S. 36. 
nommen werden sollte, belegt eine Begebenheit aus 112. Kapitel der „Älteren Hochmeisterchronik“: Der Königsberger Komtur Albrecht von Meißen zog mit seinem Heer nach Litauen. In die Zeit ihres Zuges fiel einer der vorgeschrieben Kommuniontage, an dem die Brüder die Eucharistie empfangen sollten. Dies war ihnen aber unmöglich, da sie im Feindesland waren, wo ihnen keine Kirche zur Verfügung stand, in der von den Priesterbrüdern eine Messe hätte gefeiert werden können. ${ }^{37}$ Daraufhin entfernte sich Albrecht von Meißen von seinen Truppen und betete zu Gott, dass er es ihm nicht verüble, dass er die Kommunion nicht - wie gewohnt und vorgeschrieben - empfangen könnte. Stattdessen sollte Gott seinen Willen, den Leib Christi zu empfangen, als geistiges Äquivalent zur physischen Kommunion ansehen. ${ }^{38}$ Während seines Gebetes, schwebte vom Himmel eine Hostie (der Leib Christi) auf ihn herab, die Albrecht einerseits erschrokken, andererseits froh und voller Ehrfurcht kommunizierte und Gott schließlich für die hymmelspeisze dankte. ${ }^{39}$ Aus theologischer Perspektive ist in diesem eucharistischen Speisewunder die Bestätigung der Transsubstantiationslehre zu erkennen, da die Hostie nicht als irdische, sondern als himmlische Nahrung gilt, die Albrecht auf sein Bitten hin von Gott gespendet wird. Im Kontext der Fragestellung nach der Spiritualität innerhalb des Deutschen Ordens wird hieran die von Albrecht ausgelebte intensive Begierde nach dem Kommunionempfang deutlich. Im hohen und späten Mittelalter war die tägliche oder zumindest regelmäßige Spendung der Kommunion in Brotform an Laien unüblich oder sogar untersagt, wenn nicht die Ordensregeln den Ordensangehörige im Laienstand einen häufigeren Kommunionempfang neben der beim IV. Latarankonzil 1215 für alle Gläubigen vorgeschriebenen Osterkommunion gestatteten. ${ }^{40} \mathrm{Wie}$ bereits anhand der Statuten des Deutschen Ordens dargestellt, existierten verbindliche Kommuniontage innerhalb des Ordens, die einen regelmäßigen Kommunionempfang be-

37 Die Ältere Hochmeisterchronik (wie Anm. 18), S. 581: Dy weile her was in der finde land, do gevil eyn tag, das dy brueder noch gewonheit gotis leichenam solden entphoen; das mochte em do nicht gescheen; das tet em in seynem.

38 Vgl. ebd., S. 581: Dorumb ging her weit von deme here und viel uff seyne knye, und sprach: O lieber herre Jhesu Crist, were ich hewte doheyme, so hette ich mich bereitet mit mynes gebetes ynnikeit und hette entphangen deynen werden leichenam, alse ander meyne lieben bruder thun, dorum lieber berre nym meynen willen vor dy tot.

39 Vgl. ebd., S. 581: Czu hant sach her in den louften swebyn eyne oblate kegen seynem munde. Des irschragk her sere, und was ouch froe. Dorumb sprach her: Mein lieber herre Jhesu Crist, is das deyn worer leichenam, so las en mir zcu frome komen an meyner zelen. Do methe that her den munt uff; do quam em die oblate in den munt, die her mit grosser ynnikeit entphing und danckte dem milden gote, der en mit der hymmelspeisze bedochte.

40 Vgl. P. Browe, Die Eucharistie im Mittelalter. Liturgiehistorische Forschungen in kulturwissenschaftlicher Absicht, hrsg. v. H. Lutterbach, T. Flammer, Münster ${ }^{3} 2008$, S. 39-47, 77. 
günstigten. Berichte über eucharistische Speisewunder waren im Mittelalter keine Seltenheit, oftmals wurde den Mystikern, die sich nur von der Kommunion ernährten, die Eucharistie von Vögeln herbeigebracht, dass die Hostie jedoch wie bei Albrecht zu ihm herabschwebte, war ein recht ungewöhnliches Ereignis. ${ }^{41}$ Durch die in der „Älteren Hochmeisterchronik“ geschilderte Begebenheit wird Albrecht außerdem als von Gott begnadeter Mensch ausgewiesen, da der Bitte des Ordenskomturs um Verzeihung für das Ausbleiben der Pflichtkommunion nicht nur entsprochen, sondern ihm sogar die Eucharistie gespendet wurde, die einerseits die Schuld durch die Nichteinhaltung der Regeln gegenstandslos werden ließ, andererseits im auch noch vielmehr zur Stärkung der Seele im Kampf für den christlichen Glauben dienen sollte.

\section{Christliche Allegorese von Hospitals-und Kriegsdienst}

Im übertragenen Sinne lässt sich ebenso das gemeinschaftliche Wirken in den Kontext der Spiritualität innerhalb des Deutschen Ordens eingliedern: Noch vor den liturgischen Pflichten der Ordensbrüder werden die monastischen Gelübde (Armut, Gehorsam und Keuschheit) in der 1. Regel, der Spitalsdienst im Prolog und in der 4., 5. und 6. sowie die Almosenregelung in der 7. Regel vorgeschrieben und die inhaltlichen Dimensionen dieser Dienste ausgedeutet. ${ }^{42}$ Sie spiegeln das für Ordensbrüder als ideal angesehene Verhalten und Handeln wider, bei dem sich liturgische Akte, in eime brinnendigem geiste ${ }^{43}$ ausgeübter karitativer Dienst und die militärische Verteidigung des Glaubens vereinten. ${ }^{44}$ Gabriela Wiechert fasst die spirituellen Dimensionen der drei Dienstfelder des geistlichen Rittertums zusammen und zählt die Liturgie aus ihrem Wesen heraus sowie den Hospitalsdienst als Dienst am Nächsten, der direkt in Analogie zum Matthäusevangelium (Mt 25, 40) als Gottesdienst verstanden werden konnte, zum Dienst an Christus, die Verteidigung und Verbreitung des christlichen Glaubens jedoch als Dienst für Christus. ${ }^{45}$ In diesem Spannungsfeld bewegen sich folglich die kollektiv ausgeübten Spielarten der Ordensspiritualität, von denen insbesondere die ritterlichen Pflichten in der "Älteren Hochmeisterchronik“ an vielerlei Stellen Erwähnung finden, die im Sinne einer spirituellen Relecture ebenfalls als Glaubensvollzug interpretiert werden können. Als ein Beispiel sei hierfür eine Begebenheit erwähnt, die sich laut des 67. Kapitels der Chronik auf der Burg Bartenstein in den 60er Jahren

\footnotetext{
41 Vgl. ebd., S. 113-118.

42 Vgl. Die Statuten (wie Anm. 1), S. 25 f., 29 f., 31-34.

43 Ebd., S. 26.

44 Vgl. Wiechert (wie Anm. 2), S. 137.

45 Vgl. ebd., S. 141.
} 
des 13. Jahrhunderts ereignet hat: Ein Ordensbruder bat Gott, dass er ihm weisen solle, wie nach den schweren Angriffen der Natanger fortzufahren sei. ${ }^{46}$ Daraufhin sprach eine himmlische Stimme zum Bruder, die ihm auftrug, Judea und Jerusalem für die Christen zurückzuerobern, wobei ihnen Gott helfen würde und die Ritter sich nicht fürchten sollten. ${ }^{47}$ Auch wenn es sich hier nur um eine sehr kurze Passage handelt, wird ersichtlich, dass der Kampf gegen die Heiden (hier im Heiligen Land) als direkter göttlicher Auftrag verstanden und legitimiert wurde, bei dem die Ordensbrüder auf den Beistand Gottes zählen konnten. Somit wurde der Kreuzzug von den Ordensangehörigen als Dienst für Gott und die Christenheit interpretiert, was eine spirituelle Allegorisierung dieser Rückeroberungszüge bedeutete.

\section{FaCETten DeR PRIVAT AUsgeÜbTen SPIRItUalität \\ Des Deutschen Ordens in der „Älteren Hochmeisterchronik“: Gebet und ERscheinungen}

Neben der liturgischen wird auch die mystische, private Komponente der Spiritualität innerhalb des Deutschen Ordens in zahlreichen Passagen der „Älteren Hochmeisterchronik" genau beschrieben. Hier ist nun zu unterscheiden, ob es sich um Erscheinungen, beispielsweise der Ordenspatronin Maria, um Andachtspraktiken oder um besondere Ausprägungen der Ehrerbietung gegenüber kultischen Gegenständen handelt. Die in der Quelle erwähnten Buß- und Frömmigkeitspraktiken einzelner Ordensbrüder können ebenfalls dazu dienen, die innerhalb des Deutschen Ordens ausgelebte Spiritualität zu charakterisieren. Ein Beispiel für diese geradezu körperlichen Andachtspraktiken stellt eine Begebenheit dar, die sich auf der Ordensburg Christburg ereignet haben soll: Dort lebte - laut der Schilderung im 34. Kapitel der Chronik - ein Bruder, der einen Eisengürtel auf seiner bloBen Haut trug, um Gott damit seine Liebe zu demonstrieren. ${ }^{48}$ Hierbei handelte es sich vermutlich um einen aus mehrgliedrigen eisernen Ketten gebildeten Bußgürtel, dessen Dornen sich in das Fleisch des Trägers einbohrten und der auch noch in heutiger Zeit bei verschiedenen Ordensgemeinschaften in Gebrauch ist. Die durch den Bußgürtel ausgedrückte Form der Askese besteht in der auf die paulinische Theologie (beispielsweise 1 Kor 15, 50) zurückgehende Abtötung des

46 Vgl. Die Ältere Hochmeisterchronik (wie Anm. 18), S. 567: Eyn gutter brudyr uf der burg bat unszern hirren, daz her en wold offenbarn, waz sy yn den nötin suldin thun.

47 Vgl. ebd., S. 567: Do quam vom hymmele eyne stymme; dy sprach zcu ym: Judea und Jherusalem, nicht vurchtet euch, yr sult morne von hynnen geen, und got will mit euch seyn.

48 Vgl. ebd., S. 554: Och waz zcu Cristburg eyn andir brudir yn der lybe gotis so entprand, daz her an seyner blosyn hawt trug vor eyn nachtgortel eyn grobe kethe von eysen baz an seyn ende. 
Fleisches und der aus dem Fleischlichen abgeleiteten Bedürfnisse und Triebe, um damit in ein Stadium der Selbstvergessenheit und -missachtung zu gelangen. Folglich war der in der Chronik erwähnte Ordensbruder bestrebt, alles zu unterdrükken, was ihn möglicherweise von Gott und der Konzentration auf seine Verehrung trennen könnte. In dieser Begebenheit ist jedoch noch ein weiterer Aspekt zu erkennen: Das Tragen des Bußgürtels dient in der kirchlichen Anschauung einerseits der persönlichen Kasteiung aufgrund der eigenen (leiblichen) Sündhaftigkeit und andererseits dem stellvertretenden Leiden und der daraus bestehenden körperlichen Buße für die in der Welt begangenen Sünden. ${ }^{49}$

Die größte Gruppe unter den Schilderungen dieser privaten Facette der Spiritualitätsausübung bilden die Erscheinungen, die im Folgenden genauer untersucht werden. Zunächst ist jedoch zu erörtern, worum es sich bei einer Erscheinung handelt. Peter Dinzelbacher, der sich in vielen seiner Veröffentlichungen mit diesem Thema auseinandergesetzt hat, definiert eine Vision als ein Erlebnis eines Menschen, aus seiner eigenen Umwelt an einen anderen Ort versetzt zu werden und dort einer Offenbarung teilhaftig zu werden. Dies kann in einem ekstatischen $\mathrm{Zu}$ stand oder auch im Schlaf geschehen. ${ }^{50}$ In Abgrenzung dazu handelt es sich bei einer Erscheinung um eine bildhaft beschreibbare Wahrnehmung eines Menschen, die ohne den Verlust der Wahrnehmung seiner tatsächlichen Umwelt einhergeht. Meistens erscheint dem Seher eine Person oder ein Gegenstand als wahrhaftig dort zugegen, wobei die Erscheinung den „Einbruch eines Außerweltlichen “51 darstellt, was ihr den Charakter des Übersinnlichen verleiht. Auch die Erscheinung ist häufig mit Offenbarungen oder Weisungen der erscheinenden Person verbunden. ${ }^{52}$ Ohne an dieser Stelle schon das Ergebnis der Analyse vorwegzunehmen, ist festzuhalten, dass die in der „Älteren Hochmeisterchronik“ geschilderten übersinnlichen Wahrnehmungen ausnahmslos die von Dinzelbacher eine Erscheinung definierenden Kriterien erfüllen.

Eine ganz zentrale Rolle in der Spiritualität innerhalb des Deutschen Ordens spielt Maria, die Mutter Jesu. Bernhard-Maria Rosenberg hat sich 1967 bereits ausführlich mit der marianischen Ordensfrömmigkeit beschäftigt, dabei jedoch kaum die Erscheinungsberichte in seine Überlegungen einbezogen, die hingegen im Zentrum dieses Abschnitts stehen sollen. ${ }^{53}$ Im 11. Kapitel der „Älteren Hochmeisterchronik“ wird eine Marienerscheinung geschildert, die sich auf der Burg Rehden ereignet haben soll. Der Ordensbruder, dem die Erscheinung zuteil wur-

49 Vgl. P. Lippert, Bußübungen, in: Lexikon für Theologie und Kirche (wie Anm. 4), Bd. 2, Sp. 858.

50 Vgl. P. Dinzelbacher, Vision und Visionsliteratur im Mittelalter, Stuttgart 1981, S. 29.

51 Ebd., S. 33.

52 Vgl. ebd.

53 Vgl. Rosenberg (wie Anm. 13). 
de, hegte Zweifel, ob der Deutsche Orden seine Seele retten könnte, so dass er beabsichtigte, den Orden zu verlassen, um sich einem strengeren Orden anschließen zu können. ${ }^{54} \mathrm{Im}$ Schlaf erschienen ihm die heiligen Bernhard von Clairvaux, Dominikus, Franziskus von Assisi und Augustinus, die jeweils eine Ordensregel (beziehungsweise eine normative Ordnung für ein monastisches Leben) verfasst hatten und die vom Ordensbruder um die Aufnahme in einen ihrer Orden gebeten wurden. Dies wurde ihm jedoch von allen Ordensgründern verwehrt. ${ }^{55}$ Schließlich erschien ihm im Kreis zahlreicher Brüder die Deutschordenspatronin Maria, die vom Bruder gebeten wurde, in ihrem Orden bleiben zu dürfen. Nachdem ihn Maria beschimpfte und ihm die Rückkehr verbot, sollten ihm der Deutsche Orden und seine Disziplin auch weiterhin nicht genügen, zog sie den mit ihr erscheinenden Ordensbrüdern die Mäntel aus, um dem Zweifelnden die Wunden zu zeigen, die den Brüdern im Kampf für den christlichen Glauben zugefügt worden waren. ${ }^{56}$ Durch diese Demonstration christlichen Märtyrertums und der Bereitschaft seiner Mitbrüder, für den Glauben ihr Leben hinzugeben, wurde der Bruder bekehrt und schloss sich wieder dem Orden an. Die Chronik beschreibt weiterhin, dass er sich in der Folge der Erscheinung mit noch größerer Inbrunst als zuvor Gott verschrieb und selbst für den Glauben starb, indem er von dem Prussen erschlagen wurde. ${ }^{57}$ Durch die Schilderung dieser Begebenheit wird die „Konkurrenzfähigkeit“ des Deutschen Ordens gegenüber den im Hochmittelalter gegründeten Orden wie den Franziskanern, Dominikanern, Zisterziensern oder Augustinereremiten betont: Gerade der Deutsche Orden konnte, wie aus der Chronik zu entnehmen ist, eine große Zahl an Glaubenskämpfern vorweisen, die ihr Leben bei der Verbreitung des Christentums hingaben. Außerdem diente der Deutsche Orden nicht nur der Missionierung und damit der angenommenen Seelenrettung der zu bekehrenden Heiden, sondern er führte auch die Ordensangehörigen selbst zu Gott, die durch die Aufopferung des eigenen Lebens in christlicher Anschauung ihr Seelenheil erlangen konnten. Dies unterstreicht die Verklammerung des Erscheinungsberichtes durch den anfänglichen Zweifel des Bruders an der Möglichkeit, seine Seele im Deutschen Orden zu retten zu Beginn und durch

54 Vgl. Die Ältere Hochmeisterchronik (wie Anm. 18), S. 545: Uf der selbin burg waz eyn brudyr den duchte, wy daz der dutsche ordin ym nicht mochte seyne zele yrnern. Dorum bat her orlob von seynen brudirn und wolde yn eynen strengern ordin varn.

55 Vgl. ebd., S. 545: Der brudyr bat iczlichin sundirlich, daz her en yn seyne brudyrschaft entpfinge. Dys vorsaytin sy ym alle.

56 Vgl. ebd., S. 545: Do her also stuat betrubit, do quam Maria gotis mutyr mit vil brudirn des dutschin ordins. [...] Do methe czog sy den brudirn dy mentel ab und weiste ym dy wundin und slege, do methe sy yn den tod warn gevelt um des globin willin [...].

57 Vgl. ebd.: Von dem mole vleis her sich ynniclichyr gote zcu dynen, den vor, und wart och kurezlich dornoch von den Prusyn yrslayn. 
das Martyrium desselben Bruders am Schluss dieser Erzählung. Aufgrund dieser beiden Kernaussagen wird die Instrumentalisierung des Wunderberichtes deutlich, in dem diese politischen und soteriologischen Botschaften geradezu didaktisch in das Gewand eines Erscheinungsberichtes gekleidet wurden, um darüber hinaus auch eine himmlische Bestätigung und Authentifizierung auszudrücken. Diese Anschauung wird auch in einer kurzen Begebenheit deutlich, die sich im 68. Kapitel der Chronik findet: Bei einer Schlacht gegen die Prußen verloren zahlreiche Ordensritter ihr Leben. Auf dem Schlachtfeld ließ sich ein Einsiedler nieder, der nachts an dem Ort, an dem die Ordensmitglieder im Gefecht starben, Kerzen brennen sah, was als Zeichen für das Märtyrertum und den daraus folgenden Gotteslohn angesehen wurde..$^{58}$ Das durch diese Begebenheiten vermittelte Selbstverständnis des Deutschen Ordens, der die Konkurrenz mit anderen Orden nicht scheuen muss und seine Mitglieder zum Paradies führt, wird auch an einer anderen Stelle - losgelöst von einem Erscheinungsbericht - ausgedrückt: Zusammengefasst handelt es sich um die Begegnung eines Deutschordensritters mit einem Mönch, den die Chronik als sehr korpulent beschreibt und der darüber hinaus auf einem schönen Pferd ritt. ${ }^{59}$ Es wird hieraus ersichtlich, dass dieser Mönch, der einem anderen Orden anzugehören scheint, nicht dem monastischen Ideal der Askese und Armut nacheiferte, sondern sich der Völlerei und vermutlich auch wegen des Pferdes der Eitelkeit und Prunksucht schuldig machte. Durch diese implizite Kritik wird das ritterlich-asketische Ideal des Deutschen Ordens umso mehr betont und den Brüdern zur Nachahmung empfohlen.

Eine besondere Fokussierung auf Maria besitzt auch die Episode um den Ordensbruder Hermann Sarrazin im 50. Kapitel der Chronik, der schon vor seinem Eintritt in den Orden - laut Schilderung des Chronisten - ein glühender Marienverehrer gewesen ist. In einem Kampf schlug er einen Ritter, nahm ihn als Gefangenen und verlangte ein hohes Lösegeld von ihm, um ihn nicht zu töten. Der geschlagene Ritter bat Sarrazin im Namen Mariens um seine Freilassung, die ihm daraufhin wegen seiner großen Frömmigkeit und seines Vertrauens auf Maria von Sarrazin gewährt wurde. ${ }^{60}$ Anschließend nahm Sarrazin an einem Ritter-

58 Vgl. ebd., S. 568: Uf der walstat saczte sich eyn eynszedel. Der such des nachtis gevach vil kerczin burnen uf dem velde, do dy cristin lagin yrslayn. Daz waz yo eyn czeichin, daz dy zelen, dy durch got by ledin peyn, dort von ym yr lon mit dem martyrer entphangin han.

59 Vgl. ebd., S. 558: [...] gar eyn vetter monch gar uf eym schonen pferde [...].

60 Vgl. ebd., S. 561: Der rittyr bat en durch Marien gotis muter, daz her en nicht so hog beschaczte. Do Sarracin dys horte, zcu hant sprach her zcu ym: Desze bethe vromt dyr sere, gang weg und bys aller beschatczunge vrey; P. Funk, Zur Geschichte der Frömmigkeit und Mystik im Ordenslande Preußen. Neu herausgegeben und mit Anmerkungen gemäß dem heutigen Stand der Forschung versehen von Leo Juhnke, Zeitschrift für die Geschichte und Altertumskunde Ermlands 30 (1962), 2, S. 3. 
turnier teil, nachdem er von einem Riter herausgefordert worden war, um seyner juncfrawen wille ${ }^{61}$ gegen ihn zu kämpfen. Sarrazin verstand diese Provokation als Aufforderung, für Maria bei dem Turnier anzutreten, so dass er daraufhin seinen Kontrahenten nach kurzem Kampf besiegte und den Gewinn, dy wapin mit dem pferde $e^{62}$, an arme Leute verschenkte. Nach seinem Eintritt in den Deutschen Orden legte Sarrazin jede Weltbezogenheit ab, führte ein tugendhaftes Leben und es erschien ihm oft die von ihm verehrte Ordenspatronin. ${ }^{63}$ In einer dieser Erscheinungen beschwerte sich die betrübte Ordenspatronin, dass die Ordensritter in früheren Zeiten sehr andächtig gewesen seien und bei den gemeinsamen Collationes, den abendlichen Zusammenkünften, von ihr, ihren Sohn Jesus Christus sowie die Heiligen oft gesprochen hätten, sich jedoch nun ausschließlich für weltliche Belange interessieren und von wertlichen hirren adyr bosin weibin ${ }^{64}$ sprechen würden, sie jedoch darüber völlig vergessen hätten. ${ }^{65}$

Diese beiden in der Chronik geschilderten Marienerscheinungen demonstrieren einerseits die innige Verehrung der Ordenspatronin und der Ehrerbietung ihr gegenüber innerhalb der individuellen Spiritualität der Ordensmitglieder, andererseits zeigen sie, welches geistliche und tugendhafte Ideal von geradezu höchster Instanz den Brüdern abverlangt wurde: Die Bereitschaft zum Martyrium in der konkreten Nachfolge Christi und der Mitbrüder, die bei Kreuzzügen oder heidnischen Überfällen ihr Leben verloren oder zumindest verletzt wurden, sowie die Absage an die Welt und ihre eytilkeyt ${ }^{66}$ waren in der vergeistigten Spiritualität eingeschlossen. Dass Maria in den beiden Erscheinungsberichten sehr resolut auftritt und schon fast den Charakter einer Gebieterin besitzt und in der Ordenshistoriographie dezidiert auch als kuniginne $e^{67}$ tituliert wurde, mag durch das in Riten ausgedrückte Selbstverständnis des Ordens beeinflusst worden sein, denn bei der Aufnahme in den Orden mussten die Brüder gehôrsam Gote und sente Marîen ${ }^{68}$ schwörten. ${ }^{69}$ Hierdurch wird ersichtlich, dass nicht nur die Brüder selbst, denen diese Erscheinung zuteil wurde, sondern auch diejenigen, die von der jeweilig geoffenbarten Weisung Mariens erfuhren, den Geboten Mariens Folge zu lei-

\footnotetext{
61 Die Ältere Hochmeisterchronik (wie Anm. 18), S. 561.

62 Ebd.

63 Vgl. ebd.: Dorumme geschach ys, daz Maria, gotis muter, ufte mit ym koste.

64 Ebd.

65 Vgl. ebd.

66 Ebd.

67 Vgl. Di Kronike von Pruzinlant des Nicolaus von Jeroschin, hrsg. v. E. Strehlke, in: Scriptores rerum Prussicarum (wie Anm. 18), Bd. I, Leipzig 1861, S. 19.

68 Die Statuten (wie Anm. 1), S. 128.

69 Horst (wie Anm. 32), S. 362.
} 
sten hatten. ${ }^{70}$ Philipp Funk äußerte sich in seinem Aufsatz über die mittelalterliche Frömmigkeit im Ordensland Preußen auch zur Rezeption und zum Gebrauch der historiographischen Quellen des Deutschen Ordens: Er geht davon aus, dass die Chroniken in den Remtern der Ordensburgen verlesen wurden, wodurch sie eine enorme Breitenwirkung innerhalb des Ordens erzielten. ${ }^{71}$ Aus diesem Grund sprach die in den Erscheinungen präsente Maria quasi durch die Chronik zu den Lesern oder Hörern, die ebenso wie die eigentlichen „Adressaten“ der Erscheinungen zurechtgewiesen, ermutigt oder getröstet werden sollten.

\section{Exkurs: Die marianische Spiritualität des Deutschen Ordens}

Bereits an einigen Stellen dieser Arbeit ist die besondere marianische Spiritualität und Frömmigkeit im Spiegel der „Älteren Hochmeisterchronik“ angedeutet worden. Da diese Ausprägung der Ordensspiritualität einen zentralen Stellenwert einnimmt, sollen nun einige wesentliche Punkte erläutert werden, die nicht im Erzählzusammenhang der Quelle erwähnt werden. Schon der vollständige Name „Orden der Brüder vom Deutschen Haus St. Mariens in Jerusalem“ des kurz nach dem Dritten Kreuzzug (1189-1192) gegründeten Deutschen Ordens macht die besondere Bindung an die heilige Maria deutlich. ${ }^{72} \mathrm{Die}-$ laut ordenseigener Historiographie - in Akkon versammelten Gründungsväter des Ordens knüpften mit der Namensgebung an ein 1143 erstmals belegtes, in Jerusalem von Kreuzfahrern gegründetes Hospital an, das den Beinamen „St. Marien der Deutschen in Jerusalem" trug, wobei der Verweis auf Jerusalem eine Anbindung an das Heilige Land ausdrücken sollte. ${ }^{73}$ In verschiedenen Formen artikulierten sich die Ausprägungen der marianischen Ordensspiritualität, die einerseits auch in diesem Punkt streng reglementiert war, andererseits der persönlichen Neigung unterlag, wie es das bereits vorgestellte Beispiel Hermann Sarrazins verdeutlicht hat. Wiederum war es die Liturgie, die zahlreiche marianische Gebete, Sequenzen oder Gebetszusätze, sogenannte Tropen, vorschrieb. ${ }^{74}$ Hierzu gehörten einerseits das schon erwähnte Stoßgebet Nos cum prole pia der Gesang des Salve Regina am Ende der Horen sowie marianische Erweiterungen des Kyrie- und Halleluja-Gesanges. ${ }^{75}$ Diese Zusätze verdeutlichen, dass die Angehörigen des Deutschen Ordens bei der Siche-

\footnotetext{
70 Vgl. Rosenberg (wie Anm. 13), S. 323.

71 Vgl. Funk (wie Anm. 60), S. 3.

72 Vgl. Sarnowsky (wie Anm. 26), S. 9.

73 Vgl. Rosenberg (wie Anm. 13), S. 322 f.; Sarnowsky (wie Anm. 26), S. 9, 11-14.

74 Vgl. Rosenberg (wie Anm. 13), S. 324.

75 Vgl. ebd., S. $323 \mathrm{ff}$.
} 
rung ihres Seelenheils besonders auf die Fürsprache ihrer Ordenspatronin vertrauten, der sie an verschiedenen Zeitpunkten im Gottesdienst gedachten. Hierin lässt sich auch eine Spielart des Tun-Ergehen-Zusammenhangs erkennen: Die Brüder verbreiteten den Glauben, stellten sich in den Dienst der Ordenspatronin Maria und kämpften gegen die Heiden; als eine Art „Gegenleistung“ erbaten sie für sich und ihre Angehörigen (die im Stoßgebet genannte prole) himmlischen Schutz und Erlösung. Diese Hoffnung spiegelte sich auch in zahlreichen Kirchenpatrozinien und Marienaltären der Kirchen im Ordensland sowie diversen Ortsbezeichnungen wider. ${ }^{76}$ Adam S. Labuda geht sogar so weit, die marianische Frömmigkeit als Kernmotiv der Ordensspiritualität anzusehen, die ihre besondere Manifestierung in der Kunst des Ordenslandes gefunden habe. ${ }^{77}$ Labuda verweist in diesem $\mathrm{Zu}$ sammenhang auf die Ikonographie erhaltener Altarretabel und auf die Schreinmadonnen, die als Andachtsbilder im Ordensland weit verbreitet waren. ${ }^{78}$

Interessant für den Kontext der Quelle ist die Tatsache, dass Nikolaus von Jeroschin seine Mitte des 14. Jahrhunderts entstandene ${ }^{79}$ Reimchronik zu Beginn des Werkes Maria und ihrem Lob widmete. ${ }^{80}$ Diese literarische Vorlage für die prosaische „Ältere Hochmeisterchronik“ drückt daher schon zu ihrem Beginn den besonderen Stellenwert aus, den Maria innerhalb der Chronik einnimmt, so dass den verschiedenen Schilderungen der Marienerscheinungen eine programmatische Bedeutung beigemessen werden kann. Daher sollte die Chronik vermutlich nicht nur der unterhaltsamen Belehrung und Unterweisung der Ordensbrüder dienen, was beispielsweise die leicht verständliche und gereimte Sprache nahe legt, sondern auch der Verherrlichung Mariens dienen. Die einzelnen Episoden, in denen Erscheinungsberichte enthalten sind, erhalten somit den Charakter einer Vitenfortschreibung, in denen das übersinnliche Handeln Mariens gerühmt und ihre Gegenwart im Deutschen Orden ausgedrückt wird.

\section{Handelnde Skulpturen als Zeichen des Auserwähltseins}

Eine andere, ebenfalls personale Form der Erscheinung wird ebenfalls in der „Älteren Hochmeisterchronik" beschrieben. Es handelt sich um die Verlebendigung

76 Vgl. ebd., S. 328-331.

77 Vgl. A. S. Labuda, Die Spiritualität des Deutschen Ordens und die Kunst. Der Graudenzer Altar als Paradigma, in: Die Spiritualität der Ritterorden (wie Anm. 2), Toruń 1993, S. 55.

78 Zur (marianischen) Spiritualität im Spiegel der Kunst vgl. Labuda (wie Anm. 77).

79 Vgl. Sarnowsky (wie Anm. 26), S. 83.

80 Vgl. Di Kronike (wie Anm. 67), S. 19: [...] Milde kuniginne / nû bis mîn leiterinne, want dir zu lôbis winne / diss bûchis ich beginne [...]. 
einer Skulptur in der Kapelle der Christburg, vermutlich ist es sogar in beiden Passagen dieselbe Skulptur, denn beide Begebenheiten drehen sich um ein Kruzifix, dessen Christuscorpus den vor ihm Betenden segnet oder ihm eine besondere Form der Nähe gewährt. Im 34. Kapitel der Chronik wird eine Erscheinung beschrieben, die sich an einem Karfreitag ereignet hat: Bei der Liturgie warf sich der im Rufe der heilikit ${ }^{81}$ stehende Ordensbruder von Gletzburg vor dem Kreuz nieder, um es - wie am Karfreitag üblich - mit einer Prostratio zu verehren. Während er die Wundmale des Christuscorpus küsste, löste sich ein Arm des Corpus vom Kreuz ab, um ihn zu umarmen. Doch von Gletzburg fühlte sich nicht würdig und schreckte vor der Berührung zurück. ${ }^{82}$ In derselben Kapelle betete im 45. Kapitel der Ordensbruder Heinrich Stange vor diesem Kruzifix und bat Gott um ein Zeichen, ab her seyener gnade wirdigg sei, woraufhin sich wiederum ein Arm des Gekreuzigten vom Kreuz löste und ihn segnete. Diese wundersame Begebenheit wurde nicht nur von Heinrich Stange selbst, sondern auch von einem Priesterbruder gesehen, der zur selben Zeit in der Kapelle sein Gebet verrichtete. ${ }^{84}$ Diese Ereignisse spiegeln eine zentrale Facette mittelalterlicher Spiritualität wider: Die beiden Beter sehnen sich nach größtmöglicher Nähe zu Gott und nach der Gottesschau, der visio beatifica, die sie durch die Meditation auf einer geistigen Ebene zu erreichen suchen. Gesteigert werden kann diese mentale Nähe noch durch die physische Nähe zu Gott, die beiden Brüdern vom göttlich beseelten Christuscorpus, dem lebendig gewordenen Abbild des Gekreuzigten, entgegengebracht wurde. Außerdem zeigt sich in dem Umarmungs- und Segensgestus des Kruzifixus die Wertschätzung gegenüber den beiden Ordenspersönlichkeiten, die in ihrem Lebenswandel, somit auch in ihrer Spiritualität von Gott selbst durch die Wunder bestätigt wurden. Interessanterweise haben die Berichte über diese mystischen Erfahrungen sich sogar auf die bildende Kunst auswirken können, was am folgenden Beispiel jedoch nicht mit dem Deutschen Orden in Verbindung gebracht werden

81 Die Ältere Hochmeisterchronik (wie Anm. 18), S. 554.

82 Vgl. ebd.: Undir desen waz eyner, von Gleczburg gnant, der waz so groszer heilikit; an eym karfreytage, do man beging daz ampt von unszers hirren leidunge und her mit groszer ynnikit lag vor eyme creucze, gestalt noch unszers hirren martyr, dem bilde her koste dy wunden an vusen und an henden. Seth! daz bilde holczin nam seynen arm von dem creucze und wolde den brudir ummevahin. Idoch entweich her yn demut, und sprach: O lyber hirre meyn, ich bin nicht wirdig, daz du much ummevahist.

83 Ebd., S. 560.

84 Vgl. ebd.: Der selbe Hynrich Stange knyte uf eyne czeit zcu Cristburg vor eyme altar mit andacht, und bat got, daz her ym mit eynem czeichin scheyn thete, ab her seyner gnade wirdig were. Do stunt uf dem altar eyn bilde noch unszers hirren leidunge. Daz loste seyne hant von dem cruize und gab dem brudiyr cruczewis seynen seyen. Dys sach her Hedinreich eyn pristerbrudyr, der yn der capellen yn eynem stule lag und seyn gebethe sprach. 
kann: In der Würzburger Neumünster-Kirche befindet sich ein überlebensgroßes hölzernes Schmerzensmann-Kruzifix aus dem 14. Jahrhundert, dessen Füße mit einem Nagel an den Kreuzesstamm geheftet sind, während die Arme angewinkelt sind und mit den Händen, in denen noch die Nägel stecken, den Eindruck erwecken, als wollten sie den vor der Skulptur betenden umarmen. Eine weite Verbreitung hat dieser Bildtypus nicht gefunden, doch liegt eine Inspiration durch mystische Literatur oder Erscheinungsberichte nahe (vgl. die Abbildung im Anhang). ${ }^{85}$

Eine ähnliche Nobilitierung erfuhren bei ihrer bereits angesprochenen Ordensprofess Konrad von Thüringen und die beiden Ordensbrüder Hartmann von Helderungen und Dietrich von Grüningen, die mit ihm 1234 in Marburg in den Deutschen Orden eintraten: Während der Prostratio und des Gesanges der Pfingstsequenz Veni Sancte Spiritus, do wart von al der umstendigen schar gesehin ${ }^{86}$, wie sich über den drei Professkandidaten die Flamme des auf sie herabgerufenen Heiligen Geistes befand. ${ }^{87}$ Dies verdeutlicht in der Schilderung ihr Auserwähltsein durch Gott sowie die Wahrhaftigkeit ihrer Geisterfüllung, die ihr geschicktes Agieren bei ihren Aufgaben für den Deutschen Orden schon zu Beginn ihres Wirkens sichtbar machte.

\section{GAB ES EINE CHARAKTERISTISCHE SPIRITUALITÄT INNERHALB Des Deutschen Ordens?}

Auf den ersten Blick wirkt die Spiritualität des Deutschen Ordens, wie sie sich in der "Älteren Hochmeisterchronik“ sowie in den Statuten niedergeschlagen hat, sehr breit gefächert. Auf der einen Seite stehen die ordensüblichen gemeinschaftlichen liturgischen Verpflichtungen mit einer aufgrund der Tropen starken marianischen Färbung, auf der anderen Seite wird die private vita contemplativa an vielen Stellen eingehend beschrieben. Stille, inniges Gebet vor Skulpturen mit dem Ziel der mystischen Vereinigung und Gottesschau sowie Bußübungen aus leibfeindlicher Gottesliebe werden als vorbildhaft angesehen und durch eine entsprechende Darstellung im Erzählzusammenhang der Chronik den Ordensbrüdern implizit empfohlen. Darüber hinaus wird durch die himmlische Authentifizierung durch die Gottesmutter Maria innerhalb einer Erscheinung betont, dass der vom Deutschen Orden vorgegebene und strukturierte Lebensweg, der die Möglichkeit zum

\footnotetext{
85 Vgl. Ch. Hecht, Das Schmerzensmannkreuz, in: Das Neumünster zu Würzburg. BaugeschichteRestaurierung - Konzeption, hrsg. v. J. Emmert, J. Lenssen, Regensburg 2009, S. 32-43.

86 Die Ältere Hochmeisterchronik (wie Anm. 18), S. 559.

87 Vgl. ebd.
} 
Martyrium einschließt, zum Paradies führen kann. Hierdurch wird ebenso ausgedrückt, dass der Deutsche Orden zur Konkurrenz mit zeitgenössischen (Bettel-) Orden durchaus fähig ist. Moralischen Weisungen wird innerhalb der „Älteren Hochmeisterchronik“ ebenfalls dadurch Nachdruck verliehen, indem sie ebenfalls darauf zurückgeführt werden, dass sie in einer Erscheinung von Maria geoffenbart wurden. Aufgrund des breiten Spektrums der verschiedenen Facetten der Ausübung der Spiritualität innerhalb des Deutschen Ordens im Mittelalter könnte die Schlussfolgerung lauten, dass keine charakteristische Ordensspiritualität existierte, da die in der Chronik geschilderten Ausprägungen auch in einer ähnlichen Bandbreite in der hoch- und spätmittelalterlichen Gesellschaft zu finden waren. ${ }^{88}$ Ein solches Fazit wäre jedoch zu kurz gegriffen. Zwar waren die diversen verschiedenen Artikulationsformen der Spiritualität nicht dem Deutschen Orden zueigen, die einzelne Facetten zeigten sich auch in der (Laien-)Bevölkerung, doch ist gerade das breite Spektrum, das zunächst den Eindruck einer Beliebigkeit erweckt, im Spiegel der Chronik für den Orden charakteristisch. Schließlich wurden nicht eklektisch einzelne Ausprägungen in der Spiritualitätspraxis des Ordens herausgegriffen, sondern in ihrer Allumfassendheit ausgeübt. Werden in dieses Gesamtbild schließlich auch die christozentrische Interpretation des Hospitals- und die Allegorisierung des Kriegsdienstes einbezogen, so lassen sich die Konturen der Ordensspiritualität noch schärfer nachzeichnen und von zeitgenössischen Orden abgrenzen und, gemeinsam mit der Spiritualität der Johanniter und Templer, als singulär innerhalb der mittelalterlichen Ordenslandschaft kennzeichnen.

Nun bleibt die Frage, wieso der Verfasser der „Älteren Hochmeisterchronik“ ein solch breit gefächertes Spektrum der Spiritualität innerhalb des Deutschen Ordens wiedergibt. Schon die Chronik des Peter von Dusburg kennt die verschiedenen Erscheinungs- und Wunderberichte sowie die Begebenheiten, in denen liturgische Vollzüge beschrieben werden. ${ }^{89}$ Interessanterweise ist die Schilderung dieser Begebenheiten, die auf Spiritualität zu befragende Ereignisse in den Blick nehmen, dort zu finden, wo die „Ältere Hochmeisterchronik“ sich der beiden Vorbilder, der Chroniken des Peter von Dusburg und des Nikolaus von Jeroschin, bedienen konnte, da sich die Berichte auf das 13. Jahrhundert konzentrieren. Philipp Funk mutmaßt, dass diese beiden Chronisten die geistliche Hochzeit des Ordens selbst erlebt, als Vorbild für kommende Generationen von Ordensangehörigen angesehen und daher auch pointiert nachgezeichnet hätten..$^{90}$ Im 170. Kapitel der

\footnotetext{
88 Vgl. R. Kieckhefer, Hauptströmungen der spätmittelalterlichen Frömmigkeit, in: Geschichte der christlichen Spiritualität, Bd. 2: Hochmittelalter und Reformation, hrsg. v. J. Raitt, B. McGinn, J. Meyendorff, Würzburg 1995, S. 90-123.

89 Vgl. Funk (wie Anm. 60), S. 3 f.

90 Vgl. ebd., S. 2.
} 
„Älteren Hochmeisterchronik“, nach der Vitenepisode des Hochmeisters Win-

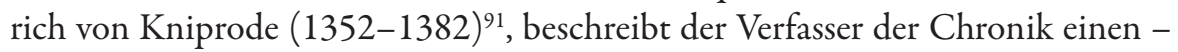
für mittelalterliche Denker - sicherlich schlüssigen Tun-Ergehen-Zusammenhang: In der Blütezeit des Ordens im 13. und frühen 14. Jahrhundert haben sich die Ordensangehörigen und ihre Hochmeister an die Gebote Gottes gehalten und waren in der Liebe zu Gott entbrannt. ${ }^{92} \mathrm{Da}$ sich die späteren Ordenspersönlichkeiten nicht mehr an den göttlichen Weisungen orientierten und Gott selbst und den Dienst für den Glauben nicht mehr in das Zentrum ihres Wirkens stellten, sandte Gott dem Land Plagen und schickte Angreifer gegen das Ordensland.93 Die mit dem Tod des Hochmeisters Winrich von Kniprode einsetzende Krise des Ordens, die schließlich in der verheerenden Niederlage in der Schlacht bei Tannenberg 1410 gipfelte, wurde somit auf Gott zurückgeführt, der die treulosen Ordensritter bestrafen und dadurch wieder zu einer wahren christlichen Grundhaltung zurückführen wollte. ${ }^{94} \mathrm{Um}$ also wieder wie in früherer Zeit gleichsam unbesiegbar zu werden, sollten sich die Verantwortlichen stärker der Gottesliebe zuwenden und die Eitelkeit der Welt hinter sich lassen. Auch rügte der Chronist die „maßlose [...] Benefizienjagd“"95, die zur Gewinnmaximierung im Namen des Glaubens betrieben wurde. ${ }^{96}$

Verbindet man nun diese Beobachtungen mit der Tatsache, dass die 1433$-1440^{97}$ verfasste „Ältere Hochmeisterchronik“ beispielsweise während der Collationes oder der gemeinsamen Mahlzeiten im Refektorium verlesen wurde, dann lässt sich der Vorbildcharakter erkennen, welcher der spirituellen Lebensweise in ihrer gesamten und im Rahmen dieser Studie geschilderten Bandbreite zugeschrieben wurde. ${ }^{98}$ Wie schon bei Peter von Dusburg und Nikolaus von Jeroschin

91 Vgl. Sarnowsky (wie Anm. 26), S. 119.

92 Vgl. Die Ältere Hochmeisterchronik (wie Anm. 18), S. 601: Do dy selben alden herren des ordens lebeten, sy hilden veste dy gebot gotis, und warn yn seyner libe heis. Dorumme wurden sy segehafft ken al iren vinden.

93 Vgl. ebd.: Abir dornoch leider begunden sy ap zcu nemen in seyner libe, und worden kallt und von tag zcu tage (leider sey is gote geclaget und seyner mutter) yo kelder an rechtem leben. [...] Dorumme vorhenget got vele pflogen obir disze armen lande, und reyset wedir sye vil finde, die sie von tag zcu tage anvechten und von jar zu jare uff das sie sich irkennen, doran sie sich nicht keren und suchen nort eyn itczlicher, was em nütcze ist, und nicht den nutcz gotis des hern Jhesu Cristi zcu irer zelen selikeit.

${ }^{4}$ Vgl. O. Engels, Zur Historiographie des Deutschen Ordens im Mittelalter, Archiv für Kulturgeschichte 48 (1966), S. 355.

95 Ebd., S. 354.

96 Vgl. ebd.

97 Vgl. ebd., S. 339.

98 Vgl. B. Schumacher, Die Idee der geistlichen Ritterorden im Mittelalter, Altpreußische Forschungen 2 (1924), S. 21. 
werden die übersinnlichen Phänomene und gemeinschaftlichen Ausübungsformen der Ordensspiritualität geschildert, jedoch in der „Älteren Hochmeisterchronik" durch die Gegenüberstellung mit dem Niedergang des Ordens im späten 14. und frühen 15. Jahrhundert umso stärker konturiert; schließlich ging - laut Schilderung des Chronisten - der militärische Zusammenbruch mit dem moralischen und spirituellen Verfall des Ordens einher. Daher könnte möglicherweise der pointierte Vergleich des früheren und zeitgenössischen Zustands des Deutschen Ordens die Brüder dazu motiviert haben, sich wieder vermehrt dem geistlichen Leben durch die Ausübung der für den Orden charakteristischen Spiritualitätsformen zu verschreiben, um damit dem machtpolitischen Niedergang entgegenzuwirken.

\section{ANHANG}

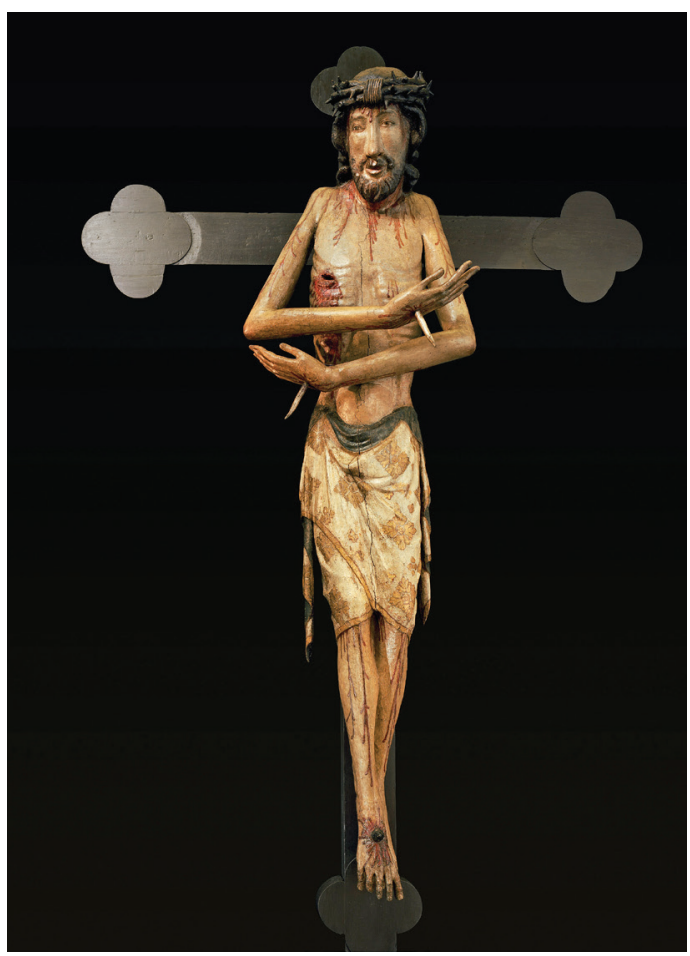

Schmerzensmannkruzifix (14. Jahrhundert), Neumünster Würzburg, Höhe $220 \mathrm{~cm}$ Abbildung: Ulrich Kneise, Eisenach. 
